\title{
Plataformas Tecnológicas e as práticas de pesquisa em biomedicina - observações preliminares sobre 0 uso de dispositivos globais e instituições locais ${ }^{12}$
}

MÁRCIA DE OLIVEIRA TEIKEIRA*

\section{Resumo}

A Política Nacional de Ciência e Tecnologia em Saúde, inspirada pela ação de organismos internacionais, como a OCDE e OMS, pela emergência das noções de inovação e de Sistema Nacional de Inovação, estimula a introdução de dispositivos de coordenação das atividades de pesquisa científica em instituições públicas de pesquisa (IPP). Assim, na última década, alguns dispositivos de coordenação foram adotados por IPPs, a exemplo das plataformas tecnológicas. Neste artigo, analisamos o uso do dispositivo "Plataforma" em um espaço local e específico de produção de ciência na área de biomedicina. Para tanto, analisamos a experiência de uma (IPP) na área de biomedicina na organização de uma Rede de Plataformas Tecnológicas. A análise concentra-se nas relações heterogêneas e

1 Trabalho apresentado no 12을 Seminário Nacional de História da Ciência e da Tecnologia / 7ํㅡㄹ Congresso Latino-Americano de História da Ciência e da Tecnologia, Salvador, 2010 no Simpósio 17 (Ciência\&Tecnologia-Sociedade-História: Abordagens construtivistas).

2 Agradeço ao Conselho Nacional de Desenvolvimento Científico e Tecnológico (CNPq) e à Fundação Carlos Chagas de Amparo à Pesquisa do Estado do Rio de Janeiro (FAPERJ) pelo apoio para a realização da pesquisa e elaboração deste artigo. Agradeço também aos professores Carlos Saldanha e Ana Filipecki pelos comentários, pelas sugestões e pela parceria em uma série de trabalhos sobre temas relacionados ao estudo das Plataformas Tecnológicas.

* Pesquisadora Adjunta da Fundação Oswaldo Cruz (FIOCRUZ) / Escola Politécnica de Saúde Joaquim Venâncio (EPSJV) - Brasil. Docente dos programas de pós-graduação em Informação e Comunicação em Saúde (Instituto de Comunicação e Informação Científica e Tecnológica em Saúde/FIOCRUZ) e Ensino de Biociências em Saúde (Instituto Oswaldo Cruz / FIOCRUZ). E-mail: marciat@fiocruz.br 
circunstanciais que sustentam a adoção e, particularmente, o uso da plataforma na coordenação de práticas de pesquisa entre grupos e laboratórios na área de biomedicina. Em síntese, este trabalho investiga o uso local de dispositivos associados à produção internacional e contemporânea de conhecimentos científicos, filiando-se, portanto, aos estudos que promovem investigações sistemáticas do processo de disseminação global de entidades.

Palavra-chave: Biomedicina. Plataformas Tecnológicas. Coalização Concorrencial. Capitalismo Acadêmico. Translocalização.

\section{Technological platforms and the biomedical research practices - preliminary notes on the use of global mechanisms and local institutions}

\section{Abstract}

The Brazilian Policy on Health Sciences and Technology, inspired both by the action of international bodies such as OECD and WHO and by the emergence of the concepts of innovation and national innovation system, encourages the introduction of mechanisms for coordinating scientific research within public research institutions (PRIs). Thus, during the last decade, the PRIs have made use of coordination mechanisms such as technology platforms. In this article I analyze the use of the mechanism "Platform" in a local and specific area of science production in the field of biomedicine. For this purpose, we analyzed the experience of a public research institution in the field of biomedicine in organizing a network of Technology Platforms. The analysis focuses on the heterogeneous and circumstantial relationships that support the adoption and particularly the use of the platform in the coordination of practices between research groups and laboratories in the area of biomedicine. In summary, this study investigates the local use of mechanisms related to the contemporary international production of scientific knowledge, thus joining the studies that promote systematic investigations on the process of dissemination of global entities.

Keywords: Biomedicine. Technology platforms. Competition coalition. Capitalism academic. Translocalization. 


\section{Introdução}

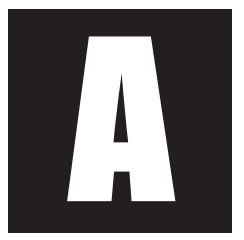

Política Nacional de Ciência e Tecnologia em Saúde, inspirada pelo ambiente de pesquisa norte-americano e da Europa Ocidental, pela ação de organismos internacionais, como a Organização de Cooperação e de Desenvolvimento Econômico (OCDE) e a Organização Mundial de Saúde (OMS), pela emergência das noções de inovação e de Sistema Nacional de Inovação no ambiente local de pesquisa, estimula a introdução de novos dispositivos de coordenação das atividades de pesquisa científica em Instituições Públicas de Pesquisa (IPP). Assim, na última década alguns dispositivos de coordenação foram adotados por IPPs, a exemplo das redes cooperativas (Teixeira et al., 2009) e das plataformas tecnológicas.

As plataformas tecnológicas, como dispositivo de coordenação, promovem a utilização em larga escala, em diferentes áreas de pesquisa e disciplinas, de equipamentos de grande porte e multiusuários associada à pesquisa científica colaborativa e à cooperação técnica entre diferentes setores econômicos. As plataformas atuam na aquisição, na manutenção e na coordenação da utilização da infraestrutura da pesquisa experimental, sobretudo desses equipamentos, por equipes de pesquisa inter ou multi-institucional (Chompalov, 1999).

No Brasil, as plataformas tecnológicas como instrumentos de coordenação e organização da pesquisa técnico-científica e de estímulo ao desenvolvimento de tecnologias remetem à ação do CNPq, mais especificamente à terceira fase do Programa de Apoio ao Desenvolvimento Científico e Tecnológico (PADCT) ${ }^{3}$. Nessa fase, decidiu-se pelo "desenho

3 Há experiências correlatas e ligeiramente anteriores de parcerias estratégicas entre instituições e organismos públicos e o setor produtivo privado, citadas por Marileusa Chiarello (2000), as Missões Tecnológicas de Minas Gerais e os Diagnósticos da demanda em setores industriais do Rio Grande do Sul, que podem ser associadas à ideia de Plataforma. 
detalhado de um processo de geração de demanda junto ao setor produtivo privado e junto aos órgãos públicos, potenciais clientes e atores na implementação de projetos cooperativos" (Rocha, 2000), incorporado ao componente de desenvolvimento tecnológico (CDT) do PADCT III. Segundo Ivan Rocha, "C. Weiss, consultor do Banco Mundial, cunhou a palavra 'plataforma' como a emulação de um ambiente de comunicação entre os atores envolvidos, tomando emprestado (por inspiração) este termo da Tecnologia da Informação" (Rocha, 2000). Marileusa Chiarello (2000), todavia, ressalta que o Subprograma de Biotecnologia do PADCT II foi pioneiro ao estimular parcerias entre atores de cadeias produtivas para a identificação de gargalos tecnológicos e resolução de problemas.

As plataformas tecnológicas do PADCT III buscavam associações entre instituições públicas de pesquisa, indústrias, serviços e organismos governamentais vinculados a um mesmo setor da economia. O intuito era identificar gargalos tecnológicos e, a partir deles, estimular a formulação de projetos cooperativos para o desenvolvimento de produtos e processos. A primeira rodada financiou uma plataforma dedicada a medicamento para uma doença negligenciada, no caso Medicamento para o combate à tuberculose proposto pela Sociedade de Química Fina para Combate a Doenças Tropicais (QTROP) (Chiarello, 2000: 99).

O propósito do PADCT, ao sugerir a adoção das plataformas, é similar aos programas financiados e/ou apoiados pela Comissão Europeia e pela OCDE. "Las Plataformas Tecnológicas son una agrupación de entidades interesadas en un sector concreto, lideradas por la industria, con el objetivo de definir una Agenda Estratégica de Investigación (..) sobre temas estratégicamente importantes y con una gran relevancia social, en los cuales lograr los objetivos europeos de crecimiento, competitividad y sostenibilidad dependen de los avances tecnológicos y de investigación a medio y largo plazo. Las Plataformas Tecnológicas se basan en la definici- 
ón de una Agenda Estratégica de investigación y en la movilización de la masa crítica de investigación y de esfuerzo innovador necesarios" (Fundación para el conocimiento, 2004). Em síntese, é possível caracterizar as plataformas tecnológicas adotadas pelo CNPq, no contexto do PADCT, e pela Comissão Europeia como instrumento para associar entidades públicas e privadas ligadas a um determinado setor produtivo e concorrendo para a definição de uma agenda estratégica de investigação (Fundación para el conocimiento, 2004).

Recentemente, o uso de plataformas tecnológicas aparece relacionado a processos de reorganização de IPPs, em especial a introdução de estruturas mais flexíveis. Todavia, há pouco conhecimento sistematizado sobre o uso desse dispositivo pelas IPPs nacionais e seus possíveis efeitos nos conhecimentos científicos produzidos.

O objetivo deste artigo, portanto, é analisar o uso do dispositivo "plataforma" em um espaço local e específico de produção de ciência na área de biomedicina ${ }^{4}$. Selecionamos, por conseguinte, a experiência de uma IPP da área de biomedicina na organização de uma rede ${ }^{5}$ de Plataformas.

O trabalho está organizado em torno de 4 seções, além desta introdução. A primeira traz considerações acerca do ambiente global de pesquisa, no qual as plataformas se disseminaram. A seguir, discorre sobre as principais técnicas e os referenciais teóricos utilizados para sistematização e análise da situação. Na terceira seção, uma descrição do ambiente local de pesquisa e das Plataformas Tecnológicas. Na quarta e última, as conclusões preliminares.

4 Ver adiante.

5 Para uma análise do uso de redes cooperativas, ver Teixeira et al. (2009; 2011). 


\section{Plataformas - Ambiente e situações de uso}

A emergência das plataformas tecnológicas, das redes cooperativas, dos consórcios de pesquisa, das alianças estratégicas e dos centros de excelência avizinha-se da disseminação da pesquisa científica colaborativa nos últimos 30 anos (Shrum et al, 2007; Chompalov et al, 1999). Na literatura, a intensificação da pesquisa colaborativa é relacionada, por sua vez, à multiplicação de projetos que requerem grandes volumes de recursos, a expertise em diferentes áreas de conhecimento, além de instrumentações complexas e diversificadas, incorporadas ou não a equipamentos (Larsen, 2008; Chompalov et al., 1999). Essas características isoladamente ou em pares se manifestam em diferentes ambientes e momentos da produção de ciência. Porém, quando associadas, descrevem a configuração contemporânea para a produção de ciência nos centros de pesquisa norte-americanos e da Europa Ocidental.

Outras características somam-se para a produção dessa configuração. As ações nacionais e internacionais de fomento à C\&T secundarizam os fundos orientados para apoiar projetos individuais de pesquisa, ancorado em um laboratório, com predomínio de uma área de conhecimento, com perspectivas difusas de interseção com setores da sociedade, em especial com a indústria. Em contrapartida, valorizam-se os fundos orientados para apoiar, fortalecer e expandir a pesquisa científica colaborativa, marcada por projetos de grande envergadura, executado por equipes de pesquisa de diferentes laboratórios (públicos e privados / industriais ou universitários), os quais suportam subcontratação de pequenas empresas, situados em regiões e países diferentes, com diversidade de áreas de conhecimento, divisão de tarefas, com perspectivas de interseção com setores específicos da sociedade bem delineados, em especial, com o setor produtivo industrial. Logo, a colaboração informal entre pesquisadores 
transfigura-se em uma forma de colaboração sustentada pelo trabalho cooperativo entre equipes de diferentes laboratórios e/ou instituições.

Todavia, duas características distinguem a atual configuração da produção de ciência - a ênfase na pesquisa de objetos e temas com alto potencial de absorção pelos setores produtivos e o uso intensivo de equipamentos de grande porte e multiusuários. Ambas estão na base da disseminação da pesquisa científica colaborativa e do uso de dispositivos de coordenação da pesquisa.

Sheila Slaughter e Gary Rhoades consideram que essa dinâmica e seus efeitos para as instituições de pesquisa e formação científica assumem a forma do "capitalismo acadêmico" (Slaughter et al., 2004). Fuller (1995) e Simon Schwartzman (1999), por sua vez, propõem pensar em um processo gradual de penetração do modo industrial na produção de conhecimento, por conseguinte, a constituição de um ambiente propício à aceleração da inovação industrial baseada em ciência por meio da aproximação e interação de centros de produção de ciência com o setor produtivo, notadamente o industrial. Nos EUA, essa aproximação conhece momentos de maior e menor intensidade, porém seu ápice é a coalização entre Estado, as universidades e centros de pesquisa com o setor industrial em torno das tecnologias militares no pós-guerra (Slaughter et al., 1996). Slaughter e Rhoades (1996; 2004), entre outros autores, elegeram o período pós-guerra fria como marco da atual configuração, na qual a coalizão em torno da guerra descola-se para a competitividade comercial entre empresas, países e bloco de países (Slaughter et al., 1996). A C\&T torna-se componente estratégico do desenvolvimento nacional, baseado no recrudescimento da competitividade econômica das corporações. Estamos, de fato, tratando da posição da C\&T na nova fase de acumulação do capital, marcada pela expansão do capital financeiro e pela reestruturação produtiva baseada amplamente na incorporação de 
novas tecnologias de produção e gestão e na flexibilização do trabalho. Trata-se de um processo de expansão da globalização da acumulação do capital e translocalização de padrões de socialização que transbordam para as práticas científicas e de organização das instituições de pesquisa (Nunes, 2002). A criação de ambientes favoráveis às interações entre universidades e centros de pesquisa acadêmica e a indústria passa por mudanças no marco regulatório, fundos de financiamento, constituição de agendas de pesquisa induzidas e pela disseminação de redes, consórcios e demais dispositivos de coordenação do trabalho de equipes dispersas em diferentes instituições em torno de um temário comum. Um elemento central do processo é o recrudescimento da ciência proprietária, traduzida pela disseminação de legislações e acordos internacionais em torno da propriedade intelectual.

No Brasil, os impactos na C\&T são sentidos com maior ênfase a partir da década de 1990 com a organização da produção, da distribuição e do consumo de bens e serviços, segundo uma estratégia mundial e para a competitividade no mercado mundial. Nesse período, além de um aumento sem precedentes dos recursos para C\&T, temos o início do processo de reformulação e posterior implantação do novo marco regulatório (Teixeira et. al. 2009), bem como de políticas setoriais, a exemplo da Política Nacional de Ciência, Tecnologia e Inovação em Saúde. A despeito do esforço nacional de afinação com as ações estratégicas e políticas globais, no tocante à C\&T o Brasil mantém algumas características singulares. A exemplo de outros países, como França (Shinn et al., 2005) e os EUA (Shapin, 2008), o Estado segue como maior financiador da atividade de pesquisa. Entretanto, as instituições e os organismos públicos são os maiores consumidores do conhecimento científico produzido. Consideramos que essa singularidade talvez influencie o modo como utilizamos localmente os dispositivos e as estratégias políticas globais de C\&T. 
Nesse ambiente de transbordamento de formas de coordenação, controle do trabalho e concepções organizacionais do mundo produtivo industrial para as instituições de C\&T, as plataformas e redes são dispositivos emergentes. Assim, quando investigamos o uso local de dispositivos associados à produção internacional e contemporânea de conhecimentos científicos, estamos reunindo elementos sobre a dinâmica local da atual configuração de produção de ciência, ou seja, sua translocalização (Nunes, 2002).

\section{Técnicas de pesquisa e referenciais teóricos}

Selecionamos a experiência da Fundação Oswaldo Cruz (Fiocruz) na organização e no uso de uma Rede de Plataformas Tecnológicas. É importante salientar que o fenômeno em estudo é um processo em desenvolvimento, assim consideramos que algumas de suas características certamente mudarão ao longo do desenvolvimento do estudo. Esse aspecto tem sido fundamental para a aproximação com os trabalhos de Howard Becker (1998) e, em especial, de Anselm Strauss (Strauss et al, 2008; 1997) sobre a estruturação e o desenvolvimento da pesquisa social. Muito embora, é importante salientar, o estudo (bem como este artigo) não possa ser tomado como um exemplo de uso da teoria ancorada (Strauss et al, 1997). É mais uma inspiração, uma lembrança permanente de como a análise dessa experiência tem exigido mudanças de rumo em função dos dados coletados e analisados concomitantemente no processo de idas e vindas a campo; assim, o quadro teórico torna-se uma versão momentânea da carta cognitiva da problemática explorada pelo pesquisador (Becker, 1998).

Com relação às técnicas, utilizamos basicamente entrevistas semiestruturadas, a observação de momentos coletivos e a análise crítica de documentos diversos e do inventário dos atores (compreendendo pesquisadores, técnicos e objetos técnicos constituintes das Plataformas). 
O estudo do uso do dispositivo "plataforma" observa princípios, proposições e preocupações do campo de Estudos Sociais da Ciência e Tecnologia (ESCT), filiando-se às problematizações do processo de disseminação global de entidades (Nunes, 2002; Mol et al, 1994), das interações entre espaços de produção de ciência e de bens (Slaughter et al., 1996, 2004; Shapin, 2008), bem como das transformações do processo de organização de produção de ciência e objetos técnicos (Law, 2002, 1994; Shapin, 2008). A análise considera as dinâmicas institucionais locais; as relações políticas, os fatores conjunturais e as injunções econômicas, o ordenamento jurídico; as práticas culturais locais; os quadros conceituais; as técnicas; os instrumentos científicos próprios a cada área de pesquisa como elementos essenciais à análise. Nesse sentido, há uma grande influência das críticas à Teoria do Ator Rede, notadamente de John Law (2002; 2004), que destacam a posição dos processos políticos e sua análise para a compreensão do processo de produção de ciência em espaços específicos.

Para analisar essa situação específica, formulamos duas questões: a) Quais processos sustentam a adoção das Plataformas?; b) Quais sentidos são atribuídos pelos atores às "plataformas tecnológicas"?

Adotamos também as seguintes premissas: primeiro, pensar nas categorias, nos conceitos e nos dispositivos como contextos específicos, nos quais as formas variam com as condições locais de produção. Tratamos os fatos, os artefatos, as plataformas e a gestão do processo de produção de conhecimentos científicos como processos situados, logo sujeitos a variações locais (Law, 2002). A análise concentra-se nas relações heterogêneas e circunstanciais que sustentam a adoção e, particularmente, o uso da plataforma na coordenação de práticas de pesquisa entre grupos e laboratórios na área de biomedicina. Segundo, se o uso desses dispositivos promove mudanças no processo de produção e difusão de conheci- 
mentos como propugna a literatura, alterações no uso poderão produzir efeitos no conteúdo e na forma dos fatos e artefatos científicos. Por outro lado, se o uso altera a forma, é possível indagar se as plataformas locais são o mesmo dispositivo que a literatura descreve e analisa ao investigar espaços de produção de ciência no centro $^{6}$ (Nunes, 2002). Assim, embora o ambiente de pesquisa apresente similaridades com os ambientes situados no centro (Nunes, 2002) e sofra as influências das atuais relações de produção de ciência, talvez elas não sejam determinantes para a configuração da forma, do conteúdo, bem como do processo de produção de conhecimentos científicos. As singularidades locais produzem deslocamentos e novas configurações (Mol et al., 1994).

Outra importante influência são as contribuições de Alberto Cambrosio e Peter Keating. Além dos trabalhos sobre plataformas (Keating et al, 2006), destacamos a noção de biomedicina empregada para designar o processo iniciado depois da Segunda Guerra Mundial de articulação entre biologia, medicina, ciência, tecnologia, inovação e rotinas. É possível abrigarmos os grupos e as instituições de pesquisa envolvidas com as grandes áreas das Ciências da Saúde e das Ciências Biológicas sob o conceito de Biomedicina, bem como temas e objetos como a pesquisa com insumos em saúde.

\section{Descrição do Ambiente de Pesquisa}

A Fiocruz é uma instituição pública centenária, vinculada ao Ministério da Saúde brasileiro e constituída por quinze Institutos distribuídos por seis cidades (Rio de Janeiro, Belo Horizonte, Curitiba, Salvador, Recife e

6 Seguindo as proposições de João Arriscado Nunes (2002) e de Xavier Polanco (1990), centro designa o conjunto de países e instituições que definem a dinâmica contemporânea da produção e difusão de conhecimentos científicos. 
Manaus) situadas em quatro diferentes regiões geográficas do país, mas com forte concentração na região sudeste. As atividades de pesquisa e desenvolvimento tecnológico concentram-se na área de biomedicina (pesquisa clínica, das ciências biológicas, das biociências) e das ciências sociais e humanas em saúde, com ênfase no estudo das doenças infecto-parasitárias (Brasil, 2007). Em 2009, a Fiocruz contabilizava 281 Grupos de Pesquisa cadastrados junto à base do Conselho Nacional de Desenvolvimento Científico e Tecnológico (CNPq), dos quais 253 na área de biomedicina.

A estrutura organizacional da Fiocruz é composta por uma Presidência (eleita para período de quatro anos e nomeada pelo Presidente da República do Brasil), quatro vice-presidências (organizadas a partir dos programas institucionais definidos no Plano Anual - PA), além de órgãos assessores. Internamente, os Institutos da Fiocruz até 2007 organizavamse em departamentos com forte presença das disciplinas científicas. Em 2007, uma nova estrutura foi aprovada, cujo foco foram a extinção gradativa da lógica departamental e o estímulo à formação de estruturas mais flexíveis (redes; plataformas de equipamentos; laboratórios flexíveis), à redução de níveis hierárquicos e à agregação de grupos em laboratórios concebidos a partir dos objetos e temas de pesquisa (Buss et al, 2002).

A pesquisa científica e o desenvolvimento tecnológico são complementados pelo ensino de pós-graduação latu e stricto sensu. A expansão do ensino iniciou-se no segundo quartel da década de 1990. Em 2001, contabilizava oito programas de pós-graduação e, em 2008, esse número atingiu o total de treze cursos (Brasil, 2009).

A Fiocruz mantém dois Institutos dedicados à produção fabril de medicamentos, vacinas e insumos diagnósticos para suprir, fundamentalmente, os programas coordenados pelo Ministério da Saúde brasileiro.

A atividade de pesquisa é financiada com recursos próprios, oriundos do orçamento do Tesouro nacional. Eles são complementados com 
fomentos individuais obtidos junto a agências nacionais e internacionais de financiamento à pesquisa, além dos recursos obtidos junto à Financiadora de Estudos e Projetos (FINEP) e ao Banco Nacional de Desenvolvimento Econômico e Social (BNDES) (Brasil, 2009).

A despeito do seu reconhecimento internacional, a Fiocruz inicia o século XXI discutindo a posição das atividades de pesquisa, desenvolvimento tecnológico e produção em seu projeto institucional. De fato, as discussões que se sucedem compartilham preocupações e temas presentes nos debates sobre o setor saúde no período. E é preciso considerar que, embora a ciência e a tecnologia (C\&T) apareçam como eixos estruturantes da política nacional de saúde desde seus primórdios, estiveram afastadas do núcleo duro da reforma sanitária. Durante uma década, houve um acentuado descolamento entre a política de saúde e a política de ciência e tecnologia (Guimarães, 2004, 2005; Alburquerque et al., 2002), descolamento agravado, em parte, pela descontinuidade de ações governamentais focadas na ciência e na tecnologia, comprometendo assim a implementação de uma política nacional e a instituição de um marco legal regulatório (Guimarães, 2005; 2004; 2003; Campos, 2005). Nos últimos 10 anos, a necessidade de conectar a pesquisa e o desenvolvimento tecnológico à política nacional de saúde ganhou expressão no setor saúde e no Governo por intermédio dos Ministérios da Saúde (MS) e da Ciência e Tecnologia (MCT). Em tela, o fortalecimento da Ciência e da Tecnologia em Saúde (CTS) como componentes da política nacional de saúde e, sobretudo, do Sistema Único de Saúde (SUS) (Guimarães, 2005; 2004).

Outro importante debate, ainda em curso, relaciona a fragilidade da base produtiva nacional em saúde com a baixa capacidade de inovação dos produtores públicos e privados. Em contrapartida, a capacidade de absorção de conhecimento científico e tecnológico pelo setor produtivo público e privado e pelos serviços de saúde também é tímida. Entre 
as consequências, estão a alta dependência da importação de produtos intensivos em conhecimentos científicos e a incipiente avaliação de tecnologias (Gadelha, 2008). A reversão dessa situação passaria, entre outras ações, pela maior articulação entre a política de saúde, a política industrial e a política de ciência e tecnologia, resultando em incentivos à pesquisa e ao desenvolvimento de tecnologias afinadas com as necessidades de saúde das populações vulneráveis (Gadelha, 2008; Buss et al., 2008; 2005; Brasil; 2007).

Cabe observar que essa última estratégia avizinha-se da diretriz da Organização Mundial de Saúde (OMS) que preconiza o aumento dos investimentos em pesquisa pelos países de menor desenvolvimento, relativo na área das doenças negligenciadas pela grande indústria farmacêutica mundial.

É nesse ambiente que a Fiocruz discute estratégias capazes de redirecionarem sua política institucional de pesquisa e de desenvolvimento tecnológico (Buss et al., 2002). O intuito dos redirecionamentos é priorizar o desenvolvimento de pesquisas de tecnologias com alto potencial de absorção pelo Sistema Único de Saúde, compreendendo os produtores nacionais de insumos em saúde públicos e privados, além dos serviços de saúde (Brasil, 2005). Assim, a partir de 2002, a Fiocruz concebe e implementa as seguintes iniciativas: Programas de Desenvolvimento Tecnológico em Insumos (PDTIS), em Saúde Pública (PDTSP), o Centro de Desenvolvimento Tecnológico em Saúde (CDTS), o reordenamento do setor de Gestão Tecnológica (GESTEC) e a estruturação de Plataformas Tecnológicas.

Nessa fase, algumas ideias que circulavam no ambiente norte-americano e europeu, notadamente nos EUA, no início dos anos 80 figuram em documentos e pronunciamentos institucionais. Nesse período, especialmente nos EUA, a ciência e tecnologia figuram entre os principais fatores para o aumento da competitividade econômica e reestruturação da base produtiva (Slaughter et al., 1996; Shapin, 2008). Os debates que 
precederam a escolha do novo presidente da Fiocruz, em 2008, oferecem alguns exemplos dessas ideias: a "saúde, a ciência e a tecnologia são instrumentos para o desenvolvimento social, para o crescimento econômico, para a geração de emprego e renda e para a democratização de oportunidades" (Gadelha, 2008); ou, ainda, a defesa da "aceleração do desenvolvimento de um ambiente favorável à inovação (...) fortalecimento da sua base produtiva e de inovação" (Gadelha, 2008).

No discurso de posse da presidência, proferido em janeiro de 2009, encontramos o reforço à tese do descolamento entre a política de saúde e a política de ciência e tecnologia - "predomina ainda, em nosso país, o fosso entre a pesquisa e a produção" (Gadelha, 2009). Em outro trecho, agora referindo-se ao lugar da Fiocruz nos próximos anos, "ser uma das âncoras do processo de desenvolvimento nacional em saúde em parceria com outras instituições nacionais e locais, ajudando a formar uma grande articulação técnica, científica, produtiva e política, para estruturar uma rede nacional de inovação voltada para as necessidades de saúde do cidadão brasileiro" (Gadelha, 2009).

Os documentos dos Congressos Internos ${ }^{7}$ da Fiocruz são outra fonte de circulação dessas ideias, a exemplo do seguinte trecho: "a saúde enquanto direito de cidadania e o desenvolvimento da base produtiva e de inovação estão intimamente relacionados. A primeira postulação, sem o suporte de uma base produtiva que lhe dê sustentação, torna-se um ideal sem materialidade. Uma base produtiva que não atenda às aspirações da melhoria da qualidade de vida e da saúde de toda a população gera iniquidade e sofrimento" (Fiocruz, 2010, p. 6). Adiante, a "saúde contribui de forma crescente para o dinamismo econômico das sociedades e sua integração com outros setores, como ciência e tecnologia, comércio

7 Ver Buss et al. (2002). 
exterior e política industrial, entre outros, pode influir decisivamente no modelo de desenvolvimento de nosso país" (Fiocruz, 2010, p. 7).

Entre 2001 e 2010, além das iniciativas identificadas, multiplicaramse pronunciamentos, documentos e ações que procuraram intensificar o desenvolvimento de tecnologias com alto potencial de absorção para o setor produtivo nacional e para o Sistema Único de Saúde (SUS). Essas ações priorizaram a inovação tecnológica diante do conjunto de atividades da instituição (Teixeira et al., 2009). Dois exemplos concretos da ação de tornar a inovação estratégica e prioritária é a organização de uma Vice-presidência de Inovação e Produção, em 2007, e do Portfólio de Inovação da Fiocruz, em meados de 2010.

\subsection{Descrição das Plataformas Tecnológicas}

No documento que instituiu o PDTIS, não há menção à constituição de uma rede de plataformas (Brasil, 2002). A iniciativa surge no processo de implementação do Programa, mas especificamente como resultado do acompanhamento e da avaliação de projetos. As Plataformas Tecnológicas foram constituídas em 2004 a partir do primeiro workshop de avaliação dos projetos apoiados pelo PDTIS. O Núcleo Gestor do PDTIS mapeou a expertise técnica na operação de equipamentos multiusuários e técnicas de base molecular disponível na Fiocruz, bem como novas necessidades frente às demandas apresentadas pelos projetos apoiados pelo Programa. Entretanto, a organização de Plataformas novas, para as quais não havia uma infraestrutura e capacidade técnica, foi posterior. Um exemplo são as Subunidades de Criopreservação e Transgenia (Plataforma de Animais de Laboratório) organizadas em 2008.

O PDTIS utiliza como dispositivo de organização a "rede cooperativa" (Teixeira et al., 2009 a,b). O mesmo dispositivo foi adotado para as Plataformas Tecnológicas. Sendo assim, a Fiocruz, por intermédio do PDTIS, possui uma Rede de Plataformas Tecnológicas. 
Embora possua uma relação umbilical com o PDTIS, a Rede de Plataformas foi concebida para funcionar segundo uma lógica de organização do trabalho bastante específica - a prestação de serviços tecnológicos para laboratórios de pesquisa na área da biomedicina. Portanto, originalmente, ela não deveria restringir-se aos projetos apoiados pelo Programa institucional de indução ao desenvolvimento tecnológico de insumo. No documento Consulta sobre utilização de Plataformas Tecnológicas à comunidade científica da Fiocruz (Brasil, 2004), lemos que: "essas plataformas tecnológicas objetivarão, pela concentração tecnológica, prestar serviços às pesquisas da Fiocruz e de outras entidades de pesquisa e desenvolvimento nacionais e eventualmente internacionais" (Brasil, 2004).

A Rede de Plataformas Tecnológicas é constituída por 12 Plataformas, subdivididas em 39 subunidades / laboratórios, distribuídas por 5 dos 6 campis da Fiocruz. Durante a realização do estudo exploratório, verificamos que há 22 subunidades instaladas no campus do Rio de Janeiro, representando 56\% do total. Em relação à sua distribuição entre os institutos técnico-científicos da Fiocruz, observamos alta concentração no Instituto Oswaldo Cruz (IOC) no Rio de Janeiro. O IOC participa de 9 plataformas, subdivididas em 17 subunidades, correspondendo a 75\% das plataformas instaladas.

As Plataformas Tecnológicas da Fiocruz foram instaladas em laboratórios de pesquisa com competência técnica na realização de ensaios e com alguma capacidade técnica para a realização de um maior volume de análises. As Plataformas Tecnológicas compartilham o espaço físico, a chefia do laboratório e, em grande parte, a mesma equipe de pesquisa dos laboratórios nos quais estão localizadas. A Fiocruz investiu no meIhoramento das instalações físicas, na aquisição de equipamentos mais modernos e/ou que possibilitam maior capacidade de processamento de amostras e ampliação da equipe técnica. 
Registramos, entretanto, uma expressiva presença de discentes vinculados aos programas de pós-graduação mantidos pela Fiocruz, notadamente de doutores, nas equipes técnicas. E há outros indícios das relações entre a Rede de Plataformas e a atividade de pesquisa - das 12 Plataformas, 10 foram instaladas em laboratórios de pesquisa. Apenas duas foram instaladas em espaços sem relação direta e imediata com a atividade de pesquisa, no caso, a Plataforma de Métodos Analíticos, instalada no Instituto de Tecnologia em Fármacos (Farmanguinhos), e a Plataforma de Animais de Laboratório, estruturada no Centro de Criação de Animais de Laboratório (CECAL) da Fiocruz.

O uso das Plataformas observa uma escala de prioridade, a saber: a) projetos apoiados pelo PDTIS; b) os demais projetos institucionais da Fiocruz; c) projetos desenvolvidos por parceiros institucionais da Fiocruz. Ainda não reunimos informações sobre como se organiza a demanda dos grupos B e C. Do mesmo modo, até o momento, não temos dados e indícios suficientes que permitam qualificar as relações entre a Rede de Plataformas e os departamentos de P\&D dos institutos de produção de vacinas e medicamento da Fiocruz. Todavia, não identificamos nenhum indício de que empresas que participam dos processos de transferência de tecnologias para os institutos estejam utilizando a Rede para realização de algum ensaio técnico.

Quanto às propriedades do ensaio, até o momento, podemos organizar as Plataformas em dois subgrupos. No primeiro grupo, estão os ensaios organizados em torno do uso intensivo de um equipamento multiusuário, a exemplo da Plataforma de Proteômica/subunidade de Espectrometria de Massa. No segundo grupo, estão os ensaios que requerem o uso de uma ou mais técnicas especializadas e que podem ou não requerer a conjugação de vários equipamentos. Como exemplo, a Plataforma de Bioensaios / subunidade Dengue. 
A coordenação da rede de plataformas, a partir de 2006, estruturou uma agenda de reuniões individuais, com gerentes de plataformas, e coletivas, com todos os gerentes. As reuniões coletivas contavam com a participação de gestores do PDTIS e da Vice-presidência de Pesquisa da Fiocruz, à qual a rede de plataformas está vinculada. O objetivo era identificar e discutir problemas e gargalos e socializar soluções encontradas pelas plataformas. Em 2009, a coordenação implantou outro dispositivo de coordenação, um workshop anual de avaliação. Além dos objetivos anteriores, o workshop envolvia a apresentação dos objetivos, a base técnica instalada e o volume de análises de cada plataforma.

\section{Considerações finais}

Este artigo participa do esforço investigativo sobre o processo de disseminação global de entidades associado à produção internacional e contemporânea de conhecimentos científicos (Nunes, 2002). Seu objetivo imediato é analisar o uso do dispositivo "plataforma tecnológica" em um espaço local e específico de produção de ciência na área de biomedicina; no caso, a experiência da Fundação Oswaldo Cruz. A caracterização do uso permite vislumbrar como a produção de ciência e dos próprios dispositivos se configura localmente. Duas questões informaram a elaboração deste artigo: a) Quais processos sustentam a adoção das Plataformas?; b) Quais sentidos são atribuídos pelos atores às "plataformas tecnológicas"?.

As plataformas da Fiocruz são espaços especializados para o desenvolvimento de ensaios tecnocientíficos. Logo, é possível compreender a rede de plataformas tecnológicas como uma rede de facilidades necessárias à P\&D de insumos em saúde. Não há assim uma grande discrepância com usos relatados pela literatura (Keating et al., 2006). 
A biomedicina como área de conhecimento é fortemente globalizada e sofre a influência de organismos internacionais comprometidos, como o que Fuller (1995) descreve como penetração do modo industrial na produção de conhecimento (Slaughter et al,. 1996). Ela também se distingue pela intensa tecnificação das práticas experimentais, pelo uso nas estratégias experimentais de equipamentos de grande porte e multiusuário, além de projetos reunindo equipes transnacionais (Shrum et al., 2007; Keating et al., 2006).

Quando analisamos a Fiocruz, identificamos estratégias que enfatizam a otimização de processos; o uso de outros dispositivos de coordenação (rede cooperativa); a ênfase em objetos com forte apelo junto ao setor produtivo em saúde, no caso tecnologias de vacinas, reativos ao diagnóstico e medicamentos.

Analisamos documentos orais e escritos que aproximam as estratégias institucionais de características da coalizão concorrencial (Slaughter et al., 1996). É possível dizer que a adoção de dispositivos (redes cooperativas e plataformas) e práticas (indução) sofre a influência desse ordenamento (coalizão concorrencial). No entanto, não é possível estimar até que ponto o processo de produção de ciência é afetado pelo uso desses dispositivos e práticas, assim como qual o peso dessa influência na conformação e operacionalização da política institucional de P\&D.

Uma forte inconformidade entre o ambiente Fiocruz e aquele identificado pela literatura como centro da constituição da plataforma como dispositivo de coordenação é a posição do setor privado. Há uma série de características que explicam essa inconformidade (Schwartzman, 1999). Destaco aqui a debilidade da P\&D no setor industrial nacional, o financiamento e a demanda da P\&D ser essencialmente públicos. É possível que essa característica afete o uso das plataformas e de outros dispositivos, 
bem como da lógica concorrencial associada ao processo contemporâneo de produção de ciência.

A literatura especializada associa algumas características e fatores com a adoção do dispositivo plataforma (Pirró e Longo et al., 2000; Chiarello, 2000; Shrum et al., 2007). A elevação dos custos com a tecnificação da pesquisa experimental (Chompalov et al., 1999; Shrum et al., 2007), além da necessidade de coordenar o trabalho de equipes espalhadas por diferentes instituições, são recorrentes em vários trabalhos (Martín-Rodríguez, 2005). Quando tentamos analisar a posição desses fatores no ambiente local da Fiocruz e correlacioná-los com a adoção da plataforma, outros elementos surgiram. A menção aos custos para manutenção e atualização do parque de equipamentos é frequente. Mas nas entrevistas e na observação das reuniões, problemas nos contratos de manutenção são insistentemente mencionados, bem como na liberação de recursos para manutenção. Contratos de prestação de serviços de manutenção e liberação de recursos nas IPPs não significam imediatamente ausência ou insuficiência de recurso, tal qual apontado pela literatura internacional especializada. Consideramos que contratos e mecanismos de operacionalização do orçamento público são especificidades locais. A questão é se as plataformas são uma estratégia viável e efetiva para contornar essas restrições.

Ainda com relação aos custos, a análise dos relatórios de gestão e atividades da Fiocruz entre 2004 e 2009 registra aumento contínuo dos recursos destinados à atividade de P\&D em biomedicina. É cedo para descartar a possibilidade do impacto dos custos de manutenção e atualização do parque de equipamentos no orçamento ser superior ao aumento dos recursos (Teixeira et al., 2009).

A coordenação de trabalho entre equipes de pesquisa localizadas em diferentes instituições não parece estar na origem da adoção pela 
Fiocruz das plataformas. Isso porque as plataformas ainda são endógenas à Fiocruz, particularmente aos projetos apoiados pelo PDTIS.

O dispositivo "Plataformas Tecnológicas" em geral está associado à prestação de serviços técnicos. No caso da Fiocruz, julgamos que a institucionalização da atividade de prestação de serviços é mais um dos elementos do processo de centralização da inovação. Porém, passados sete anos, a efetivação do projeto de institucionalizar uma base para prestação de serviços tecnológicos é um objetivo não alcançado. A Rede de Plataformas é bastante exitosa ao propiciar o acesso de projetos do PDTIS, de discentes de pós-graduação e pesquisadores da Fiocruz a facilidades tecnocientíficas na área de biomedicina. Todavia, não há indícios de que essa rede de facilidades atenda a projetos extramuros, nacionais ou internacionais. Ao menos, não de forma consistente. Há duas possíveis explicações. Primeiro, a necessidade de ampliação da capacidade de operação das plataformas. Segundo, talvez a ampliação da prestação de serviços tecnológicos seja um projeto em disputa ou ao menos não completamente consensual. Nesse sentido, é interessante observar que quando contrapomos a escala de prioridade de projetos para uso das plataformas ao documento Consulta sobre utilização de Plataformas Tecnológicas à comunidade científica da Fiocruz (Brasil, 2004), as prioridades apontam "projetos desenvolvidos por parceiros institucionais da Fiocruz", enquanto o documento de 2004 é bem mais amplo ("outras entidades de pesquisa e desenvolvimento nacionais e eventualmente internacionais"). É certo que as prioridades podem mudar em função da maior ou menor capacidade de operação das plataformas, todavia elas indicam as normas que operam e organizam o cotidiano da rede de plataformas. 


\section{Referências}

ALBUQUERQUE, E. da M. E.; CASSIOLATO, J. E. As especificidades do Sistema de Inovação do Setor Saúde. Revista de Economia Política, Rio de Janeiro, 22 (4), p. 134-15, 2002.

BRASIL. Ministério da Saúde. Fundação Oswaldo Cruz. PDTIS. Rio de Janeiro: Fiocruz. 84 p. 2002. Mimeo.

BRASIL. Ministério da Saúde. Fundação Oswaldo Cruz. Consulta sobre utilização de Plataformas Tecnológicas à comunidade científica da Fiocruz. 2004. Mimeo.BUSS, P. M.; GADELHA, P. Fundação Oswaldo Cruz - experiência centenária em biologia e saúde pública. São Paulo em Perspectiva, v.16, n.4. p.73-83, 2002. Disponível em: <http://www.scielo.br/pdf/spp/v16n4/13578.pdf>.

CALLON, M.; LARÉDO, P.; MUSTAR, P. La gestion stratégique de la recherche et de la tecnologie. L'évaluation des programmes. Paris: Economica, 1995.

CHIARELLO, Marileusa. As Plataformas tecnológicas e a promoção de parcerias para a inovação. Parcerias Estratégicas, n. 8, p. 93 - 102, 2000.

CHOMPALOV, Ivan; SHRUM, Wesley. Institutional Collaboration in Science: a Typology of Technological Practice. Science, Technology \& Human Values, Vol. 24, no 3, p. 338-372, 1999.

FIOCRUZ. Documento Base do VI Congresso Interno da Fiocruz. 2010. Disponível em:<http://www.fiocruz.br/congressointerno/media/documento_congresso_interno_01092010\%5B1\%5D.pdf>. Acesso em: 02 de setembro de $\overline{2} 010$.

GADELHA, Carlos G. Complexo Econômico-Industrial da Saúde: uma visão geral. In: SEMINÁRIO SOBRE COMPLEXO ECONÔMICO-INDUSTRIAL DA SAÚDE, Rio de Janeiro, BNDES / Ministério da Saúde, 2008. Mimeo.

GADELHA, Paulo. Programa de Campanha. 2008. Mimeo.

GADELHA, Paulo. Discurso de Posse. 2009. Disponível em: http://www.fiocruz. $\mathrm{br} / \mathrm{ccs} / \mathrm{cgi} / \mathrm{cgilua}$. exe/sys/start.htm? infoid=2273\&sid=9. Acesso em: 02 de setembro de 2010.

GUIMARÃES, R. F. Avaliação de tecnologia em saúde. Palestra proferida no II Seminário sobre o Complexo Industrial da Saúde, Rio de Janeiro, BNDES, 29 de setembro de 2003.

GUIMARÃES, R. F. Bases para uma política nacional de ciência, tecnologia e inovação em saúde. Ciência \& Saúde Coletiva, Rio de Janeiro, 9 (2), p. 375-387, 2004. 
GUIMARÃES, R. F. Pronunciamento de Abertura. In: 2ª CONFERÊNCIA NACIONAL DE CIÊNCIA, TECNOLOGIA E INOVAÇÃO EM SAÚDE. Anais... Brasília: Ministério da Saúde, 2005.

KEATING, P.; CAMBROSIO, A. Biomedical Platforms: realigning the normal and the pathological in late-twentieth-century medicine. Cambridge, MA: The MIT Press, 2006. 560 p.

LARSEN K. Knowledge network hubs and measures of research impact, science structure and publication output in nanostructured solar cell research. Scientometrics, 74 (1), p. 123-142, 2008.

LAW, John. Aircraft Stories: decentering the object in technoscience. Duke: Duke University Press, 2002. 264 p.

LAW, John. Organizing modernity. Oxford. Blackwell Publishing, 1994. 219 p.

LAW, John; HASSARD, John. Actor network theory and after. Oxford: Blackwell Publishing, 2004. 257 p.

MARTÍN-RODRÍGUEZ, L. S.; BEAULIEU, M. D.; D'AMOUR, D.; FERRADA-VIDELA, M. The determinants of successful collaboration: A review of theoretical and empirical studies. Journal of Interprofessional Care, Supplement 1, p.132-147, 2005.

MOL, A.; LAW, J. Regions, networks and fluids: anaemia and social topology. Social Studies of Science, 24 (4), p. 641-672, 1994.

NUNES, João Arriscado. As dinâmicas da(s) ciência(s) no perímetro do centro: Uma cultura científica de fronteira. Revista Crítica de Ciências Sociais, 63, p. 189-198, 2002.

PIRRÓ E LONGO W.; OLIVEIRA, A. R. P. Pesquisa Cooperativa e Centros de Excelência. Parcerias Estratégicas, 9, p. 129-144, 2000.

POLANCO, Xavier. Une science-monde: la mondialisation de la science européenne et la création de traditions scientifiques locales. In: POLANCO, Xavier (Org.). Naissance et développment de la science-monde. Paris: La Decouverte, 1990.

ROCHA, Ivan. Plataforma: conceito e operação. Disponível em: http://www. abipti.org.br/plataforma2/pla_ivanrocha.htm. Acesso em: 02 de setembro de 2010.

SHAPIN, Steve. The scientific entrepreneur, money, motives and the place of virtue. In: SHAPIN, Steve. The Scientific life: a moral history of a late modern vocation. Chicago; London: The University of Chicago Press, 2008. Chapter 7. p $209-267$. 
SHINN, Terry; RAGOUET, P. Controverses sur la science. Pour une sociologie transversaliste de l'activité scientifique. Paris: Raisons d'Agir Éditions, 2005.

SHRUM, W.; GENUTH, J.; CHOMPALOV, I. Structures of Scientific Collaboration. Cambridge: The MIT Press, 2007. 296p

SLAUGHTER, Sheila; RHOADES, Gary. The emergence of a competitiveness research and development policy coalition and the commercialization of academic science and technology. Science, Technology, and Human Values, 21, p 303-39, 1996.

SLAUGHTER, Sheila; RHOADES, Gary. Academic capitalism and the new economy: markets, state, and higher education. The Johns Hopkins University Press, 2004.

STRAUSS, A; CORBIN, J. Grounded theory in practice. Thousand Oaks, CA: Sage, 1997.

STRAUSS, A; CORBIN, J. Pesquisa Qualitativa. Técnicas e Procedimentos para o Desenvolvimento de Teoria Fundamentada. Porto Alegre: Artmed Editora, 2008. $288 p$

TEIXEIRA, M. O.; MACHADO, C. J. S; FILIPECKI, A. T. P.; KLEIN, H. E. A dinâmica da organização da pesquisa em biomedicina no Brasil: anatomia de uma experiência recente na Fundação Oswaldo Cruz. RECIIS, 3 (2), p 1-26, 2009. Disponível em: htttp://www.reciis.cict.fiocruz.br/index.php/reciis/article/view/255/277.

TEIXEIRA, Marcia O.; MACHADO, C. J. S; FILIPECKI, A. T.; Descrição e análise do uso de um instrumento de coordenação em um instituto público de pesquisa em biomedicina. Ciência \& Saúde Coletiva [online], vol.16, n.3, p. 1835-1847, 2011.

Recebido em: 29/03/2011

Aceite final: $30 / 08 / 2011$ 
